Spin Physics (SPIN2014)

International Journal of Modern Physics: Conference Series

Vol. 40 (2016) 1660070 (4 pages)

(C) The Author(s)

DOI: 10.1142/S2010194516600703

\title{
Study of Three-Nucleon Force Effect via Few-Nucleon Scattering
}

\author{
Yasunori Wada, Kimiko Sekiguchi, Uwe Gebauer, Jumpei Miyazaki, Takahiro Taguchi \\ Department of Physics, Tohoku University, Sendai, Miyagi, 980-8578, Japan \\ wada@lambda.phys.tohoku.ac.jp \\ Masanori Dozono, Hideyuki Sakai, Naruhiko Sakamoto, Masaki Sasano, Yohei Shimizu, \\ Hiroshi Suzuki, Tomohiro Uesaka \\ Nishina Center, RIKEN, Wako, Saitama, 351-0198, Japan \\ Shoichiro Kawase, Yuki Kubota, Cheong Soo Lee, Tsz Leung Tang, Kentaro Yako \\ Center for Nuclear Study, University of Tokyo, Tokyo, 113-0033, Japan \\ Yukie Maeda \\ Fuculty of Engineering, University of Miyazaki, Miyazaki, Miyazaki, 889-2192, Japan \\ Kenjiro Miki, Hiroyuki Okamura \\ Research Center for Nuclear Physics, Osaka University, Ibaraki, Osaka, 567-0047, Japan \\ Satoshi Sakaguchi, Tomotsugu Wakasa \\ Department of Physics, Kyusyu University, Higashi, Fukuoka, 812-8581, Japan
}

Published 29 February 2016

\begin{abstract}
A complete set of deuteron analyzing powers for elastic deuteron-proton $(d p)$ scattering at 250 and $294 \mathrm{MeV} /$ nucleon has been measured. The obtained data are compared with the Faddeev calculations based on the modern nucleon-nucleon forces together with threenucleon forces.
\end{abstract}

Keywords: Three-nucleon force; ${ }^{1} \mathrm{H}(\vec{d}, d){ }^{1} \mathrm{H}$; Deuteron analyzing powers.

\section{Introduction}

Understanding the nuclear properties from bare nuclear forces is one of the main topic in nuclear physics. Recently the importance of three-nucleon forces (3NFs), which appear when more than two nucleons interact, has been indicated in various

This is an Open Access article published by World Scientific Publishing Company. It is distributed under the terms of the Creative Commons Attribution 3.0 (CC-BY) License. Further distribution of this work is permitted, provided the original work is properly cited. 
nuclear phenomena, such as few-nucleon scattering, binding energies of nuclei, ${ }^{1}$ and equation of state of nuclear matter. ${ }^{2}$

Three-nucleon scattering at intermediate energies $(\gtrsim 60 \mathrm{MeV} /$ nucleon $)$ is one attractive approach to investigate the dynamical aspects of $3 \mathrm{NFs}$, such as momentum dependence and/or spin dependence. For few-nucleon scattering rigorous numerical calculations in terms of Faddeev theory by using bare nuclear potentials have been realized. Direct comparison between those calculations and precise data enables us to discuss on the effects of 3NFs quantitatively.

With the aim of clarifying roles of $3 \mathrm{NFs}$ in nuclei, the experimental programs with polarized deuteron beams at intermediate energies are in progress at RIKEN RI beam factory (RIBF) ${ }^{4-6}$ Here, we report recent results of the measurement of complete set of deuteron analyzing powers $\left(A_{y}^{d}, A_{y y}, A_{x x}, A_{x z}\right)$ for $d p$ elastic scattering at 250 and $294 \mathrm{MeV} /$ nucleon. $^{6}$

\section{Experiment}

The schematic view of the experimental setup is shown in Fig. 1. At RIBF, the vector and tensor polarized deuteron beams were provided by the polarized ion source $^{3}$ and accelerated by the injector cyclotron AVF and RIKEN Ring cyclotron (RRC) up to 90 (100) $\mathrm{MeV} /$ nucleon, and then up to 250(294) $\mathrm{MeV} /$ nucleon by the superconducting Ring cyclotron (SRC). The beam polarizations were monitored by the polarimeter Dpol prior to acceleration by the SRC. The measurement for $d p$ elastic scattering was carried out using the polarimeter BigDpol installed at the extraction beam line of the SRC. The beams were stopped by the Faraday cup shown

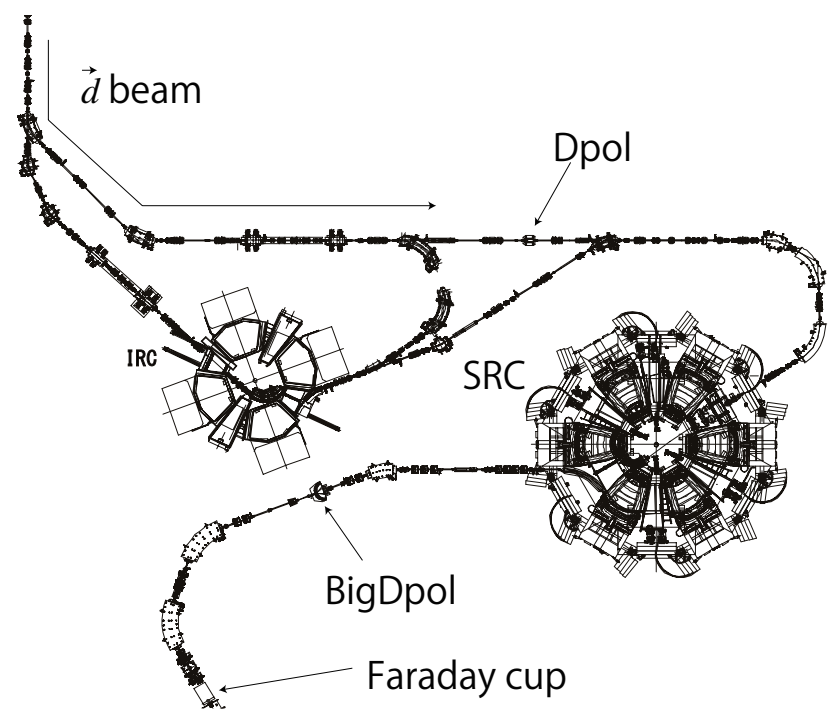

Fig. 1. A schematic view of the experimental setup for measurement of $d p$ elastic scattering at RIKEN. 
in Fig. 1. A polyethylene $\left(\mathrm{CH}_{2}\right)$ film with the thickness of $330 \mathrm{mg} / \mathrm{cm}^{2}$ was used as a hydrogen target, and four pairs of plastic scintillators coupled with photomultiplier tubes were mounted in two independent planes, $90^{\circ}$ apart in azimuthal angle, and operated in kinematic coincidence for elastic $d p$ scattering. The measured angles in the center of mass system are $\theta_{\text {c.m. }}=40^{\circ}-160^{\circ}$. The direction of beam polarization axis was controlled by the spin rotator Wien filter prior to acceleration. Single turn extraction features of RIBF cyclotrons made it possible to maintain the polarization amplitudes during acceleration. The beam polarizations were $80 \%$ of theoretical maximum values during the experiment.

\section{Result}

Figure 2 shows newly obtained experimental data of all deuteron analyzing powers $\left(A_{y}^{d}, A_{y y}, A_{x x}, A_{x z}\right)$ at 250 and $294 \mathrm{MeV} /$ nucleon together with the previously reported data at $135 \mathrm{MeV} /$ nucleon. ${ }^{7}$ Open circles are the data with statistical errors. The data are compared with the Faddeev calculations based on the modern NN forces together with the 3NFs. The red(blue) bands in the figure are the Faddeev calculations with(w/o) the Tucson-Melbourne'99 (TM99) $3 \mathrm{NF}^{8}$ based on the modern NN potentials, namely CDBonn, ${ }^{9}$ AV $18,{ }^{10}$ Nijmegen I and II. ${ }^{11}$ The solid lines are the calculations with the Urbana IX $3 \mathrm{NF}^{12}$ based on the AV18 potential.

At a lower energy $135 \mathrm{MeV} /$ nucleon, vector analyzing power $i T_{11}$ is well described by adding $3 \mathrm{NFs}$. On the other hands, for $T_{22}$, the calculation based on NN forces only already shows a moderate agreement with the data. Besides, the
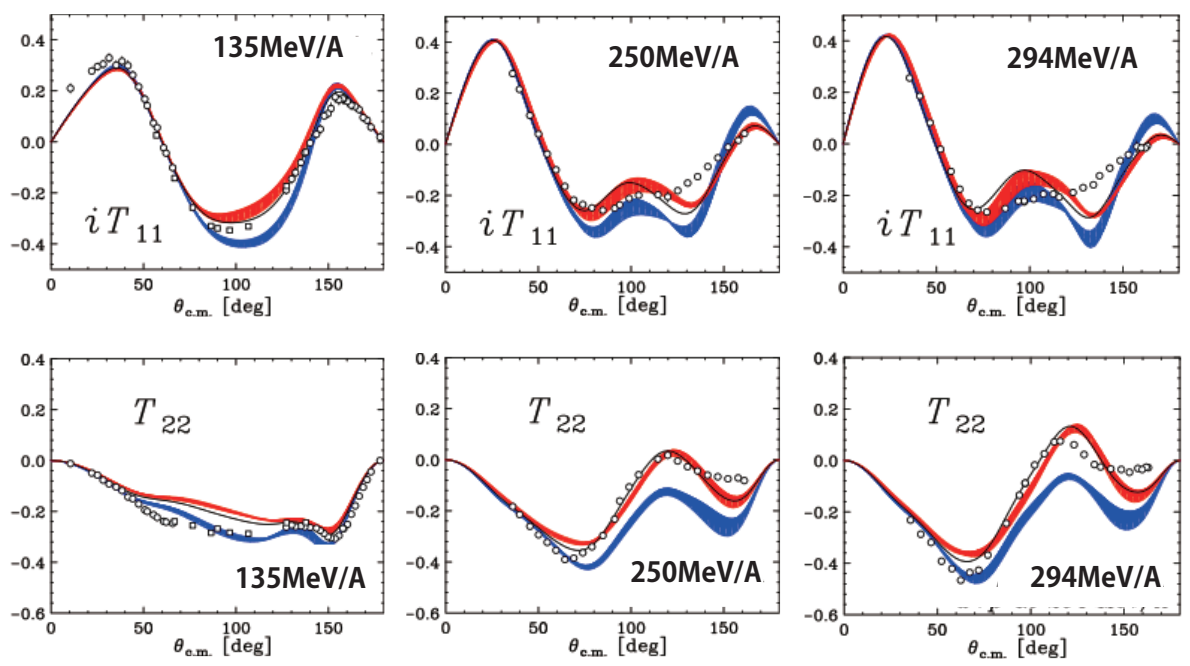

2NF(CD Bonn, AV18, Nijimegen I,II)

Fig. 2. Deuteron analyzing powers $i T_{11}, T_{22}$ for elastic $d p$ scattering at $135-294 \mathrm{MeV} /$ nucleon. 
3NFs effects cause the discrepancies between the data and the calculation. At higher energies 250 and $294 \mathrm{MeV} /$ nucleon, different results are obtained from those at $135 \mathrm{MeV} /$ nucleon in comparison between the data and the calculations. At these higher energies, all deuteron analyzing powers are well described by the calculation adding 3 NFs at forward angles $\left(\theta_{\text {c.m. }} \lesssim 120^{\circ}\right)$. However, at backward angles larger than $120^{\circ}$, the effects of $3 \mathrm{NFs}$ provided by current models do not explain the difference between the data and the calculations based on NN forces. The relativistic effects are estimated to be small for these polarization observables for $d p$ elastic scattering. ${ }^{6}$ Therefore, the important components of the $3 \mathrm{NF}$ are missing in the calculations, e.g. short range parts of three-nucleon forces.

\section{Summary}

We have performed the experiment of $d p$ elastic scattering at 250 and $294 \mathrm{MeV} /$ nucleon and measured the complete set of deuteron analyzing powers with high precision. In comparison between the data and the calculations presented here significantly different results are obtained at 250 and $294 \mathrm{MeV} /$ nucleon from those at a lower energy (135 MeV/nucleon). To improve the description of the data at our energies, short-range 3NF components should be applied in the calculations.

\section{References}

1. S. C. Pieper et al., Phys. Rev. C 64, 014001 (2003).

2. A. Akmal et al., Phys. Rev. C 58, 1804 (1998).

3. H. Okamura et al., AIP Conf. Proc. 293, 84 (1994).

4. K. Sekiguchi et al., Phys. Rev. C 65, 034003-1 (2002).

5. K. Sekiguchi et al., Phys. Rev. C 83, 061001-1 (2011).

6. K. Sekiguchi et al., Phys. Rev. C 89, 034003-1 (2014).

7. H. Sakai et al., Phys. Rev. Lett. 84, 5188 (2000).

8. S. A. Coon and H. K. Han, Few Body Syst. 30, 131 (2001).

9. R. Machleidt, Phys. Rev. C 63, 024001 (2001).

10. R. B. Witinga et al., Phys. Rev. C 51, 38 (1995).

11. V. G. J. Stoks et al., Phys. Rev. C 49, 2950 (1994).

12. B. S. Pudliner et al., Phys. Rev. C 56, 1720 (1997). 